International Journal of Pure and Applied Mathematics

Volume 108 No. 4 2016, 781-790

ISSN: 1311-8080 (printed version); ISSN: 1314-3395 (on-line version)

url: http://www.ijpam.eu

doi: 10.12732 /ijpam.v108i4.4

\title{
GEOMETRIC PROPERTIES FOR INTEGRO-DIFFERENTIAL OPERATOR INVOLVING THE PRE-SCHWARZIAN DERIVATIVE
}

\author{
Zainab E. Abdulnaby ${ }^{1,2} \S$, Adem Kilicman ${ }^{1}$, Rabha W. Ibrahim ${ }^{3}$ \\ ${ }^{1}$ Department of Mathematics \\ Faculty of Science \\ Universiti Putra Malaysia \\ 43400 UPM, Serdang, Selangor, MALAYSIA \\ ${ }^{2}$ Department of Mathematics \\ College of Science \\ Al-Mustansiriyah University \\ Baghdad, IRAQ \\ ${ }^{3}$ Faculty of Computer Science and Information Technology \\ University Malaya \\ 50603, MALAYSIA
}

\begin{abstract}
Recently, the study of operators theory (differential, integral, integro-differential) has been increased. It appears widely in the geometric function theory, to create some generalized subclasses of analytic functions. In this effort, we introduce a generalized integrodifferential operator $\mathfrak{J}_{m}(\mathbf{z})$ and obtain its properties by utilizing the pre-Schwarzian derivative. Applications are illustrated, based on fractional calculus in the sequel.
\end{abstract}

AMS Subject Classification: 30C45, 30C55

Key Words: fractional calculus, analytic functions, unit disk, univalent functions, fractional differential operator, integral operator

\section{Introduction}

The geometric function theory is very extensive area to involve various kinds

Received: March 26, 2016

Published: August 16, 2016

$\S$ Correspondence author (c) 2016 Academic Publications, Ltd. url: www.acadpubl.eu 
of operators. These operators are playing an important role in geometric function theory to impose new generalized subclasses of analytic univalent and then study their properties. By utilizing the method of convolution, Salagean [1], Introduced the differential operator $\mathcal{D}_{n}$. Tracked by Al-Oboudi differential operator [2]. Several authors have applied the Salagean operator to impose and consider the properties of certain known and new classes of analytic univalent functions. We mention at this juncture some of them in recent years. Najafzadeh [3], studied a new subclass of analytic univalent functions with negative and fixed coefficient based on Salagean and Ruscheweyh differential operators. Aouf et al. [4], provided some consequences for certain subclasses of analytic functions founded by the concept of Salagean operator with varying arguments. El-Ashwah [5], introduced Salagean operator to define a new subclass of analytic functions and derived some subordination results for this subclass in open unit disk. Breas [6], explored a new general integral operator for certain holomorphic functions created by the Salagean differential operator and studied some properties for this integral operator on some subclasses of univalent function. Similarly, Deniz [7], defined a new general integral operator by considering the Hadamard product and gave new sufficient conditions for this operator to be univalent in $\mathbb{U}$. Breaz el at. [8], demarcated two general integral operators $F_{\lambda}(z)$ and $G_{\lambda}(z)$ and investigated some geometric properties for these operators on subclasses of analytic function in open unit disk. More investigations can be found in $[9,10,11]$.

The pre-Schwarzian and the Schwarzian derivatives of locally univalent analytic mappings $f$ are extensively recommended implements in the investigation of geometric properties of such functions. For occurrence, it can be utilized to develop either necessary or sufficient conditions for the global univalence, or to get confident geometric conditions on the range of $f$ (see [12]). In this study, we investigate to give the best estimate for the norm of pre-Schwarzian derivative of integro-differential operator $\mathfrak{J}_{n}(z)$ in open unit disk.

\section{Preliminaries}

let $\mathcal{H}$ be the family of holomorphic functions on open unit disk $\mathbb{U}=\{z:|z|<1\}$ of complex $z$-plane $\mathbb{C}$ and let $\mathcal{A}$ denote the class of functions of the form

$$
f(z)=z+\sum_{m=2}^{\infty} a_{m} z^{m}
$$


which are analytic in the open unit disk $\mathbb{U}$ and usually normalized by

$$
f(0)=f^{\prime}(0)-1=0 .
$$

Let denoted by $\mathcal{S}$ the subclass of $\mathcal{A}$ consisting of functions $f$ of form (1) which are univalent function. For a positive real number $\psi$ with $0 \leq \psi<1$, a function $f \in \mathcal{A}$ is said to be starlike, if

$$
\operatorname{Re}\left\{\frac{z f^{\prime}(z)}{f(z)}\right\}>\psi, \quad(z \in \mathbb{U}) .
$$

and we denoted by $\mathcal{S}^{*}(\psi)$ the class of starlike functions of order $\psi$. Further $f$ is said to be convex if

$$
\operatorname{Re}\left\{\frac{z f^{\prime}(z)}{f(z)}+1\right\}>\psi, \quad(z \in \mathbb{U}) .
$$

also, we denoted by $\mathcal{K}(\psi)$ the class of convex functions of order $\psi$ where $0 \leq$ $\psi<1$. Note that

$$
J[f] \in \mathcal{K}(\psi) \Longleftrightarrow f(z) \in \mathcal{S}^{*}(\psi)
$$

where $J[f]$ is Alexander transform [13] of $f \in \mathcal{A}$ defined by

$$
J[f](z)=\int_{0}^{z} \frac{f(\eta)}{\eta} d \eta=\int_{0}^{1} f(t z) \frac{d t}{t} .
$$

Let $f: \mathbb{U} \rightarrow \mathbb{C}$ be analytic and locally univalent holomorphic function $f(z)$. The pre-Schwarzian derivative $T_{f}(z)$ of $f$ is the logarithmic derivative of $f^{\prime}$. That is defined by

$$
T_{f}(z)=\frac{f^{\prime \prime}(z)}{f^{\prime}(z)} .
$$

The Schwarzian derivative of such a function $f$ is defined by

$$
S_{f}=\left(T_{f}\right)^{\prime}-\frac{1}{2}\left(T_{f}\right)^{2}
$$

More specifically, it is well known that any univalent analytic transformation $f$ in the unit disk satisfies the sharp inequality

$$
\left|T_{f}(z)\right| \leq \frac{6}{1-|z|^{2}}, \quad z \in \mathbb{U}
$$

For a locally univalent of $f \in \mathbb{U}$, the norm of $T_{f}$ is given by 


$$
\left\|T_{f}\right\|=\sup _{|z|<1}\left(1-|z|^{2}\right)\left|\frac{f^{\prime \prime}(z)}{f^{\prime}(z)}\right|
$$

As is well known this norm is considered by Becker [14] (Becker's univalence criterion). The following results can be located in $[15,16,17]$ :

Theorem 1. Let $f$ be analytic and locally univalent in open unit disk $\mathbb{U}$, we obtain:

(i) If $\left\|T_{f}\right\| \leq 1$, then $f$ is univalent in $\mathbb{U}$, and

(ii) If $\left\|T_{f}\right\| \leq 2$, then $f$ is bounded.

In terms of the Schwarzian derivative, we have the sharp inequality

$$
\left|T_{f}(z)\right| \leq\left(\frac{6}{\left(1-|z|^{2}\right.}\right)^{2}, \quad z \in \mathbb{U}
$$

Theorem 2. For $f \in \mathcal{A}$ and $0 \leq \psi<1$, we obtain:

(i) If $f$ is starlike function of order $\psi$ then $\left\|T_{f}\right\| \leq 6-4 \psi$.

(ii) If $f$ is convex function of order $\psi$ then $\left\|T_{f}\right\| \leq 4(1-\psi)$.

The second tool, in this investigation is the integro-differential operator. The authors interested a differential operator of two parameters in [18] and defined by, let $\mathcal{T}^{\mu, \gamma}: \mathcal{A} \rightarrow \mathcal{A}$,

$$
\mathcal{T}^{\mu, \gamma} f(z):=z+\sum_{m=2}^{\infty} \frac{\Gamma(\gamma+1)}{\Gamma(\mu+1)} \frac{\Gamma(m+\mu)}{\Gamma(m+\gamma)} a_{m} z^{m} . \quad z \in \mathbb{U}
$$

for some $0<\mu \leq 1,0<\gamma \leq 1$ and $m \in \mathbb{N} \backslash\{0,1\}$. When $\mu=\gamma$ in (4), then we obtain

$$
\mathcal{T}_{z}^{\mu, \gamma} f(z)=f(z)
$$

for more details (see [19]).

Now, let define a fractional differential operator $D_{\delta}^{k}: \mathcal{A} \rightarrow \mathcal{A}$ as follows:

$$
\begin{aligned}
D_{\delta}^{0} f(z) & =f(z), \\
D_{\delta}^{1} f(z) & =(1-\delta) \mathcal{T}^{\mu, \gamma} f(z)+\delta z\left(\mathcal{T}^{\mu, \gamma} f(z)\right)^{\prime} \\
\quad & \\
D_{\delta}^{k} f(z) & =D_{\delta}\left(D_{\delta}^{k-1} f(z)\right) \quad k \in \mathbb{N} .
\end{aligned}
$$


with $D_{\delta}^{k}(0)=0$. In general we write

$$
D_{\delta}^{k} f(z)=z+\sum_{m=2}^{\infty} \Psi_{m, k}(\mu, \gamma, \delta) a_{m} z^{m} \quad k \in \mathbb{N}_{0}=\{0,1,2, \ldots\} .
$$

where

$$
\Psi_{m, k}(\mu, \gamma, \delta)=\left[\frac{\Gamma(\gamma+1)}{\Gamma(\mu+1)} \frac{\Gamma(m+\mu)}{\Gamma(m+\gamma)}(1+(m-1) \delta)\right]^{k}
$$

Remark 1. For $k \geq 0$ and $0<\gamma \leq 1,0<\mu \leq 1$, we see that

(i) when $\mu=\gamma, \delta=1$ and $k=1$, we reduce to Salagean operator [1] and

(ii) when $\mu=\gamma$, we reduce to Al-Oboudi differential operator [2].

Now, we investigate some new subclasses of $\mathcal{A}$ define as follows:

Let define the class $\mathcal{S}^{k}(\delta, \phi)$ by

$$
\operatorname{Re}\left\{\frac{z D_{\delta}^{k+1} f(z)}{D_{\delta}^{k} f(z)}\right\}>\phi, \quad z \in \mathbb{U}
$$

where $0 \leq \phi<1, \delta \leq 0, k \in\{0,1, \cdots\}$ and let define the class $\mathcal{K}^{k}(\delta, \phi)$ by

$$
\operatorname{Re}\left\{\frac{z D_{\delta}^{k+2} f(z)}{D_{\delta}^{k+1} f(z)}+1\right\}>\phi, \quad z \in \mathbb{U}
$$

for some $0 \leq \phi<1, \delta \leq 0$ and $k \in\{0,1, \cdots\}$. If $k=0$ in (9) and (10), then we have the well known function classes

$$
\mathcal{S}^{0}(\delta, \phi)=\mathcal{S}^{*}(\phi), \quad 0 \leq \phi<1
$$

and

$$
\mathcal{K}^{0}(\delta, \phi)=\mathcal{K}(\phi), \quad 0 \leq \phi<1
$$

Recently, Stanciu and Breaz in [11] proposed the general integral operator

$$
\mathcal{G}_{n}(z)=\int_{0}^{z} \prod_{i=1}^{n}\left(\frac{f_{i}(t)}{t}\right)^{\lambda_{i}}\left(g_{i}^{\prime}(t)\right)^{\nu_{i}} d t
$$

where $\lambda_{i}$ and $\nu_{i}$ are two real numbers, $i=1,2, \cdots, n$. and $n \in \mathbb{N}$.

Now, we purpose to define a new generalized integral operator which derive from the integral operator (11) with the utility of the fractional differential operator in (7). 
Definition 3. Let the generalized integral operator $\mathfrak{J}_{n}(z): \mathcal{A}^{n} \rightarrow \mathcal{A}$ defined by

$$
\mathfrak{J}_{n}(z)=\int_{0}^{z} \prod_{i=1}^{n}\left(\frac{D_{\delta}^{k} f_{i}(t)}{t}\right)^{\lambda_{i}}\left(D_{\delta}^{k+1} g_{i}(t)\right)^{\nu_{i}} d t
$$

where $f_{i}, g_{i} \in \mathcal{A}, \lambda_{i}, \nu_{i}$ be positive real numbers in $\mathbb{R}, i=1,2, \cdots, n$ and $n \in \mathbb{N}$.

Remark 2. Note that the integral operator $\mathfrak{J}_{n}(z)$ is generalized for the following operators introduced and investigated by several authors:

(i) For $k=0$, we have integral operator defined in (11), which studied by Stanciu and Breaz in [11].

(ii) For $k=0$ and $f_{i}^{\prime}=g_{i}^{\prime} ; i=1,2, \cdots, n$, we have integral operator

$$
\mathcal{F}_{\nu, \lambda} f(z)=\int_{0}^{z} \prod_{i=1}^{n}\left(f_{i}^{\prime}(t)\right)^{\nu_{i}}\left(\frac{f_{i}(t)}{t}\right)^{\lambda_{i}} d t .
$$

was studied and considered by Frasin in [20].

(iii) For $k=0$ and $\lambda_{i}=0 ; i=1,2, \cdots, n$, we have integral operator

$$
\mathcal{I}_{n}(z)=\int_{0}^{z} \prod_{i=1}^{n}\left(\frac{f_{i}^{\prime}(t)}{t}\right)^{\nu_{i}} d t .
$$

considered by D. Breaz and N. Breaz in [9].

(iv) For $k=0$, and $\lambda_{i}=0 ; i=1,2, \cdots, n$, we have the integral operator

$$
\mathcal{I}_{\nu_{n}}(z)=\int_{0}^{z} \prod_{i=1}^{n}\left(g_{i}^{\prime}(t)\right)^{\nu_{i}} d t .
$$

which studied by Breaz et al. [10]. In particular, for $\nu_{1}=\nu$ and $g_{1}=g$, we have the integral operator

$$
\mathcal{I}_{\nu} f(z)=\int_{0}^{z}\left(g^{\prime}(t)\right)^{\nu} d t
$$

which was studied by Kim et al. in [17].

(v) For $k=0, n=1, \nu_{1}=0, \lambda_{1}=\lambda$ and $f_{1}=f$, we have operator

$$
\mathcal{I}_{\lambda} f(z)=\int_{0}^{z}\left(\frac{f(t)}{t}\right)^{\lambda} d t .
$$

investigated in [21]. In particular, for $\lambda=1$, we have Alexander integral operator given by (2). 


\section{Main Results}

We start our first outcome

Theorem 4. Let $\nu_{i}, \lambda_{i}$ be positive real numbers, $i=1,2, \cdots, n$. If $f_{i}, g_{i} \in$ $\mathcal{K}^{k}(\delta, \phi)$ and $h_{i} \in \mathcal{S}^{k}(\delta, \phi)$. Assume that $\mathfrak{J}_{n}(z)$ is locally univalent in $\mathbb{U}$, if

$$
\left\|T_{g_{i}}\right\| \leq \frac{1}{2}\left(\sum_{i=1}^{n} \nu_{i}\right)^{-1} \quad\left\|T_{h_{i}}\right\| \leq \frac{1}{2}\left(\sum_{i=1}^{n} \lambda_{i}\right)^{-1}
$$

then $\mathfrak{J}_{n}(z)$ is univalent. Moreover, if

$$
\left\|T_{g_{i}}\right\| \leq\left(\sum_{i=1}^{n} \nu_{i}\right)^{-1} \quad\left\|T_{h_{i}}\right\| \leq\left(\sum_{i=1}^{n} \lambda_{i}\right)^{-1}
$$

then $\mathfrak{J}_{n}(z)$ is bounded.

Proof. Since

$$
\begin{aligned}
\left\|T_{\mathfrak{J}_{n}}\right\| & =\sup _{|z|<1}\left(1-|z|^{2}\right)\left|\frac{\mathfrak{J}_{n}^{\prime \prime}(z)}{\mathfrak{J}_{n}^{\prime}(z)}\right| \\
& =\sup _{|z|<1}\left(1-|z|^{2}\right)\left|\frac{\left(\int_{0}^{z} \prod_{i=1}^{n}\left(\frac{D_{\delta}^{k} f_{i}(t)}{t}\right)^{\lambda_{j}}\left(D_{\delta}^{k+1} g_{i}(t)\right)^{\nu_{i}} d t\right)^{\prime \prime}}{\left(\int_{0}^{z} \prod_{i=1}^{n}\left(\frac{D_{\delta}^{k} f_{i}(t)}{t}\right)^{\lambda_{i}}\left(D_{\delta}^{k+1} g_{i}(t)\right)^{\nu_{i}} d t\right)^{\prime}}\right|
\end{aligned}
$$

then we calculate the derivative of the first and second order of integral operator $\mathfrak{J}_{n}(z)$ on norm defined by $(3)$, we obtain

$$
\begin{aligned}
& \left\|T_{\mathfrak{J}_{n}}\right\|=\sup _{|z|<1}\left(1-|z|^{2}\right) \mid \frac{\nu_{1}\left[\mathcal{D}_{\delta}^{k+2} g_{1}(z)\right]\left[\mathcal{D}_{\delta}^{k+1} g_{1}(z)\right]^{\nu_{1}-1}\left(\frac{\mathcal{D}_{\delta}^{k} f_{1}(z)}{z}\right)^{\lambda_{1}}}{\left[\mathcal{D}_{\delta}^{k+1} g_{1}(z)\right]^{\nu_{1}}\left(\frac{\mathcal{D}_{\delta}^{k} f_{1}(z)}{z}\right)^{\lambda_{1}}} \\
& \times \frac{\left[\mathcal{D}_{\delta}^{k+1} g_{2}(z)\right]^{\nu_{2}}\left(\frac{\mathcal{D}_{\delta}^{k} f_{2}(z)}{z}\right)^{\lambda_{2}} \times \cdots \times\left[\mathcal{D}_{\delta}^{k+1} g_{n}(z)\right]^{\nu_{n}}\left(\frac{\mathcal{D}_{\delta}^{k} f_{n}(z)}{z}\right)^{\lambda_{n}}}{\left[\mathcal{D}_{\delta}^{k+1} g_{2}(z)\right]^{\nu_{2}}\left(\frac{\mathcal{D}_{\delta}^{k} f_{2}(z)}{z}\right)^{\lambda_{2}} \times \cdots \times\left[\mathcal{D}_{\delta}^{k+1} g_{n}(z)\right]^{\nu_{n}}\left(\frac{\mathcal{D}_{\delta}^{k} f_{n}(z)}{z}\right)^{\lambda_{n}}} \\
& +\frac{\lambda_{1}\left(\frac{\mathcal{D}_{\delta}^{k} f_{1}(z)}{z}\right)^{\prime}\left(\frac{\mathcal{D}_{\delta}^{k} f_{1}(z)}{z}\right)^{\lambda_{1}-1}\left[\mathcal{D}_{\delta}^{k+1} g_{1}(z)\right]^{\nu_{1}}\left[\mathcal{D}_{\delta}^{k+1} g_{2}(z)\right]^{\nu_{2}}\left(\frac{\mathcal{D}_{\delta}^{k} f_{2}(z)}{z}\right)^{\nu_{2}}}{\left[\mathcal{D}_{\delta}^{k+1} g_{1}(z)\right]^{\nu_{1}}\left(\frac{\mathcal{D}_{\delta}^{k} f_{1}(z)}{z}\right)^{\lambda_{1}}\left[\mathcal{D}_{\delta}^{k+1} g_{2}(z)\right]^{\nu_{2}}\left(\frac{\mathcal{D}_{\delta}^{k} f_{2}(z)}{z}\right)^{\lambda_{2}}} \\
& \times \frac{\cdots\left[\mathcal{D}_{\delta}^{k+1} g_{n}(z)\right]^{\nu_{n}}\left(\frac{\mathcal{D}_{\delta}^{k} f_{n}(z)}{z}\right)^{\lambda_{n}}}{\cdots\left[\mathcal{D}_{\delta}^{k+1} g_{n}(z)\right]^{\nu_{n}}\left(\frac{\mathcal{D}_{\delta}^{k} f_{n}(z)}{z}\right)^{\lambda_{n}}}+\cdots \mid
\end{aligned}
$$


then,

$$
\begin{aligned}
\left\|T_{\mathfrak{J}_{n}}\right\|= & \sup _{|z|<1}\left(1-|z|^{2}\right) \times \mid \nu_{1} \frac{\mathcal{D}_{\delta}^{k+2} g_{1}(z)}{\mathcal{D}_{\delta}^{k+1} g_{1}(z)}+\lambda_{1} \frac{\left(\frac{\mathcal{D}_{\delta}^{k} f_{1}(z)}{z}\right)^{\prime}}{\left(\frac{\mathcal{D}_{\delta}^{k} f_{1}(z)}{z}\right)}+\cdots \\
& \quad+\nu_{n} \frac{\mathcal{D}_{\delta}^{k+2} g_{n}(z)}{\mathcal{D}_{\delta}^{k+1} g_{n}(z)}+\lambda_{n} \frac{\left(\frac{\mathcal{D}_{\delta}^{k} f_{n}(z)}{z}\right)^{\prime}}{\left(\frac{\mathcal{D}_{\delta}^{k} f_{n}(z)}{z}\right)} \mid \\
= & \sup _{|z|<1}\left(1-|z|^{2}\right)\left|\sum_{i=1}^{n} \nu_{i} \frac{\mathcal{D}_{\delta}^{k+2} g_{i}(z)}{\mathcal{D}_{\delta}^{k+1} g_{i}(z)}+\sum_{i=1}^{n} \lambda_{i} \frac{\left(\frac{\mathcal{D}_{\delta}^{k} f_{i}(z)}{z}\right)^{\prime}}{\left(\frac{\mathcal{D}_{\delta}^{k} f_{i}(z)}{z}\right)}\right|,
\end{aligned}
$$

by using Alexander's theorem we have

$$
f_{i} \in \mathcal{K}^{k}(\delta, \phi)
$$

and $h \in \mathcal{S}^{k}(\delta, \phi)$ then

$$
\begin{gathered}
f_{i}(z)=z h_{i}^{\prime}(z) . \\
\left\|T_{\mathfrak{J}_{n}}\right\|=\sup _{|z|<1}\left(1-|z|^{2}\right)\left|\sum_{i=1}^{n} \nu_{i} \frac{\mathcal{D}_{\delta}^{k+2} g_{i}(z)}{\mathcal{D}_{\delta}^{k+1} g_{i}(z)}+\sum_{i=1}^{n} \lambda_{i} \frac{\mathcal{D}_{\delta}^{k+2} h_{i}(z)}{\mathcal{D}_{\delta}^{k+1} h_{i}(z)}\right| \\
\leq \sup _{|z|<1}\left(1-|z|^{2}\right)\left|\sum_{i=1}^{n} \nu_{i} \frac{\mathcal{D}_{\delta}^{k+2} g_{i}(z)}{\mathcal{D}_{\delta}^{k+1} g_{i}(z)}\right|+\sup _{|z|<1}\left(1-|z|^{2}\right)\left|\sum_{i=1}^{n} \lambda_{i} \frac{\mathcal{D}_{\delta}^{k+2} h_{i}(z)}{\mathcal{D}_{\delta}^{k+1} h_{i}(z)}\right| .
\end{gathered}
$$

Hence,

$$
\left\|T_{\mathfrak{J}_{n}}\right\| \leq \sup _{|z|<1}\left(1-|z|^{2}\right)\left|\sum_{i=1}^{n} \nu_{i} \frac{\mathcal{D}_{\delta}^{k+2} g_{i}(z)}{\mathcal{D}_{\delta}^{k+1} g_{i}(z)}\right|+\sup _{|z|<1}\left(1-|z|^{2}\right)\left|\sum_{i=1}^{n} \lambda_{i} \frac{\mathcal{D}_{\delta}^{k+2} h_{i}(z)}{\mathcal{D}_{\delta}^{k+1} h_{i}(z)}\right| .
$$

So, we have

$$
\left\|T_{\mathfrak{J}_{n}}\right\| \leq \sum_{i=1}^{n} \nu_{i}\left\|T_{g_{i}}\right\|+\sum_{i=1}^{n} \lambda_{i}\left\|T_{h_{i}}\right\|
$$

Theorem 5. Let $f_{i}, g_{i}, h_{i}, i=1,2, \cdots, n$ be a family of analytic functions where $f_{i}$ and $g_{i}$ are convex of order $\phi_{i}, i=1,2, \cdots, n$ and $h_{i}$ are starlike of order $\phi_{i}, i=1,2,3, \cdots, n$. Then, we have

$$
\left\|T_{\mathfrak{J}_{n}}\right\| \leq \sum_{i=1}^{n} \nu_{i}\left(4-4 \phi_{i}\right)+\sum_{i=1}^{n} \lambda_{i}\left(6-4 \phi_{i}\right)
$$


Proof. From Theorem 2 and expression (18) we obtain the proof of Theorem 5.

By setting $k=0$ in Theorem 5, we have

Corollary 6. Let $f_{i}, g_{i}, h_{i}, i=1,2,3, \cdots, n$ be a family of analytic functions where $f_{i}$ and $g_{i}$ are convex of order $\phi$ and $h_{i}$ are starlike of order $\phi$ then, we have

$$
\left\|T_{\mathfrak{J}_{n}}\right\| \leq(4-4 \phi) \sum_{i=1}^{n} \nu_{i}+(6-4 \phi) \sum_{i=1}^{n} \lambda_{i} .
$$

By setting $k=0$ and $n=1$ in Theorem 5, we obtain

Corollary 7. Let $f, g$ and $h$ be analytic functions where $f$ is convex of order $\psi$ and $g$ is starlike of order $\phi$, if $\nu_{1}=\nu$ and $\lambda_{1}=\lambda$ then

$$
\left\|T_{\mathfrak{J}_{n}}\right\| \leq(4-4 \phi) \nu+(6-4 \phi) \lambda
$$

\section{Conclusion}

We introduced an integro-differential operator defined by two types of operators in the unit disk. We have seen that the operator is convex as well as bounded under some conditions. The main strategy is the per-Schwarzian derivative. This kind of derivative plays an important role to estimate classes in the geometric function theory.

\section{References}

[1] G.S. Salagean, Subclasses of univalent functions, In Complex Analysis-Fifth Romanian-Finnish Seminar, Springer Berlin Heidelberg, 1013 (1983), 362-372, doi: 10.1007/BFb0066543.

[2] F.M. Al-Oboudi, On univalent functions defined by a generalized Salagean operator, International Journal of Mathematics and Mathematical Sciences, 2004, No. 27 (2004), 1429-1436, doi: 10.1155/s0161171204108090.

[3] S. Najafzadeh, Application of Salagean and Ruscheweyh operators on univalent holomorphic functions with finitely many coefficients, Fractional Calculus and Applied Analysis, 13, No. 5 (2010), 517-520.

[4] M.K. Aouf, R.M. El-Ashwah, A.A.M. Hassan and A.H. Hassan, On subordination results for certain new classes of analytic functions defined by using Salagean operator, Bulletin of Mathematical Analysis and Applications, 4, No. 1 (2012), 239-246, doi: 10.1016/j.jmaa.2004.08.066. 
[5] R.M. El-Ashwah, Subordination results for certain subclasses of analytic functions defined by Salagean operator, Acta Universitatis Apulensis, No. 37 (2014), 197-204.

[6] D. Breaz, H.O. Güney, and G.S. Salagean, A new general integral operator, Tamsui Oxford Journal of Mathematical Sciences, 25, No. 4 (2009), 407-414.

[7] E. Deniz, D. Răducanu, and H. Orhan, On the univalence of an integral operator defined by Hadamard product, Applied Mathematics Letters, 25, No. 2 (2012), 179-184, doi: 10.1016/j.aml.2011.08.011.

[8] D. Breaz, S. Owa, and N. Breaz, Some properties for general integral operators, Advances in Mathematics, 3, No. 1 (2014), 9-14.

[9] D. Breaz and N. Breaz, Two integral operators, Studia Universitatis Babes-Bolyai, Mathematica, 47, No. 3 (2002), 13-19.

[10] D. Breaz, S. Owa and N. Breaz, A new integral univalent operator, Acta Universitatis Apulensis, 16 (2008), 11-16.

[11] L. Stanciu, D. Breaz, Some properties of a general integral operator, Bulletin of the Iranian Mathematical Society, 40, No. 6 (2014), 1433-1439.

[12] P. Duren, Univalent Functions, Springer-Verlag, New York, (1983).

[13] J.W. Alexander, Functions which map the interior of the unit circle upon simple regions, The Annals of Mathematics, 17, No. 1 (1915), 12-22, doi: 10.2307/2007212.

[14] J. Becker, Löwnersche Differentialgleichung und quasikonform fortsetzbare schlichte Funktionen, Journal für die reine und angewandte Mathematik, 255 (1972), 23-43, doi: $10.1515 /$ crll.1972.255.23

[15] Y.C. Kim, S. Ponnusamy, and T. Sugawa, Geometric properties of nonlinear integral transforms of certain analytic functions, Proceedings of the Japan Academy, Series A, Mathematical Sciences, 80, No. 5 (2004), 57-60, doi: 10.3792/pjaa.80.57.

[16] J.H. Choi, Y.C. Kim, S. Ponnusamy, and T. Sugawa, Norm estimates for the Alexander transforms of convex functions of order alpha, Journal of mathematical analysis and applications, 303, No. 2 (2005), 661-668, doi: 10.1016/j.jmaa.2004.08.066.

[17] Y.C. Kim, S. Ponnusamy, and T. Sugawa, Mapping properties of nonlinear integral operators and pre-Schwarzian derivatives, Journal of mathematical analysis and applications, 299, No. 2 (2002), 433-447, doi: 10.1016/j.jmaa.2004.03.081.

[18] Z. Esa, A. Kilicman, R.W. Ibrahim, M.R. Ismail, and S.S. Husain, Application of Modified Complex Tremblay Operator, Proceeding of 2nd International Conference on Mathematical Sciences and Statistics (ICMSS2016), Kuala Lumpur, Malaysia, (2016), in press.

[19] Z. Esa, H.M. Srivastava, A. Kilicman, and R.W. Ibrahim, A Novel Subclass of Analytic Functions Specified by a Family of Fractional Derivatives in the Complex Domain, Filomat, (2016), in press.

[20] B.A. Frasin, Univalence criteria for general integral operator, Mathematical Communications, 16, No. 1 (2011), 115-124.

[21] S.S. Miller, P. Mocanu, M.O. Reade, Starlike integral operators, Pacific Journal of Mathematics, 79, No. 1 (1978), 157-168, doi: 10.2140/pjm.1978.79.157. 